DOI: $10.14361 / 9783839446195-011$

\title{
Kreativität zur Partizipationsförderung
}

\author{
Der Ansatz einer Bildung zur Innovativität
}

\section{Claudia Scharf, Inga Gryl, Swantje Borukhovich-Weis, Benjamin Rott}

Die Gesellschaft strebt nach (technischem) Fortschritt und nach kreativen Ideen, die zu diesem Fortschritt verhelfen sollen. ${ }^{1}$ Eine solche neoliberale Betrachtungsweise von Kreativität ist insofern problematisch, als sie den Begriff seines (gesellschafts-)kritischen Potenzials beraubt und reale Partizipation (vgl. Hart 1992) unterbindet. Dieser Beitrag strebt an, dieses Dilemma aufzulösen, indem er den Ansatz einer Bildung zur Innovativität vorstellt, welcher auf eine Befähigung zu realer Partizipation abzielt. Einen integralen Bestandteil dieses Ansatzes bildet Kreativität in einer humanistischen Lesart nach Popitz (2000). Die theoretische Auseinandersetzung mit diesem Kreativitätskonzept soll eine Grundlage schaffen, schulische Methoden auszuloten, welche eine Bildung zur Innovativität ermöglichen.

\section{(Kreativitäts-)Bildung in ihrer neoliberalen Kontextualisierung}

Gegenwärtige westliche Gesellschaften sind deutlich durch neoliberale Praktiken geprägt. Sie basieren auf dem Primat der Marktökonomie, was weitreichende Folgen mit sich bringt (vgl. Boas/Gans-Morse 2009; Springer/Birch/MacLeavy 2016). Der Neoliberalismus wurde begünstigt durch spezifische technische und gesellschaftliche Veränderungen: Als Antworten auf den verschärften internationalen Wettbewerb im Zuge der Globalisierung werden u.a. die flexible Spezialisierung sowie die Maximierung der betrieblichen Reaktionspotenziale gegeben. Dies erfordert neue Strategien der Arbeitskraftnutzung, wobei die Selbstorganisation innerhalb der neoliberalen Zielstellungen zum Systemerhalt an die Stelle direkter,

1 Wie z.B. das Ringen um einen hohen Platz in der Rangliste des Global Innovation Index (Cornell University/INSEAD/WIPO 2018) indiziert (vgl. u.a. „Weltweiter Vergleich: Deutschland ist am innovativsten“). 
aber unflexibler Weisungen tritt. So erfolgt ein systematischer Zugriff auf Sozialund Kommunikationskompetenzen, Begeisterungsfähigkeit, Leistungsbereitschaft, Loyalität und Solidarität des*der Einzelnen (vgl. Sennett 1998) und schlussendlich die Individualisierung und Internalisierung externer Ziele, was mit dem Begriff des „Unternehmer[s] seiner selbst“ (Bröckling 2004: 239) erfasst wird. Jene Neoliberalisierung geschieht eher verborgen als offensichtlich (vgl. Gille 2013), ,[v]ielmehr lassen sich Normalisierungs- und Naturalisierungstendenzen beobachten“ (ebd., 87). Dieses Nicht-Wissen um die eigene Instrumentalisierung, welche Žižek $(2012)^{2}$ im Bildungskontext als subtile Ideologisierung beschreibt, erschwert demokratisches und widerständiges Agieren (vgl. ebd. sowie Freire 1970; Macedo 2006; Gille 2013).

Im Zuge dieser Entwicklung ist zu erkennen, dass auch der Kreativitätsbegriff instrumentalisiert wird (vgl. Raunig/Wuggenig 2007): Output- und Marktorientierung kapitalistischer Gesellschaftsformen inkorporieren den durch von Osten (2003) benannten Imperativ ,Be creative!', nach welchem Individuen stetig zu sog. kreativem Handeln aufgefordert sind. ${ }^{3}$ Der Imperativ ist jedoch weniger auf kreatives Handeln im Sinne einer gestalterisch-(gesellschafts-)kritischen Tätigkeit, denn vielmehr auf ökonomisch orientiertes Handeln ausgerichtet (vgl. von Osten 2003), bei dem nur die Erneuerung den langfristigen Erhalt von Marktmacht sichert (vgl. Schumpeter 2005). Wer die eigene Kreativität nicht nutzt, scheint sich nicht richtig in die (Markt-)Gesellschaft einzubringen und nicht über Resilienz (vgl. Braun $2011)^{4}$, Originalität, Hingabefähigkeit und Frustrationstoleranz zu verfügen, die eine sog. „kreative Persönlichkeit“5 (Holm-Hadulla 2005: 33) ausmachen. Dies

2 Dabei scheinen Žižeks (2012) Ausführungen auf Althussers (2010) Aufsatz zu ,Ideologie‘, ,Ideologischen Staatsapparaten' und dem ,Repressiven Apparat‘ zu basieren.

3 Zur Umdeutung von Kreativität im Rahmen unternehmerischer Steuerungsmodi und für Beispiele, damit verbundenen Anforderungen im Arbeitsalltag widerständig zu begegnen, vgl. den Beitrag von Sarah Nies in diesem Band.

4 Kritisch zum Resilienzbegriff vgl. „Resilienz \& neoliberale ,Eigenverantwortung““.

5 Holm-Hadulla (2005: 45ff.) beschreibt die Eigenschaften und Fähigkeiten einer ,kreativen Persönlichkeit“ mit dem Akronym „FASZINATION“ (ebd.): Flexibilität (nur bei Stabilität in Politik, Wirtschaft und Wissenschaft möglich), Assoziatives Denken (Perspektivenwechsel, aber keine „richtungslose Träumerei“ [ebd., 47]), Selbstvertrauen (Resilienz, Selbstmanagement, Habitus), Zielorientierung, Intelligenz (in Kombination mit Offenheit), Nonkonformismus (Skepsis gegenüber Konventionen), Authentizität (Selbstverantwortung, abzugrenzen von ,unproduktiver Eigenbrötelei“ [ebd., 51]), Transzendenz (sich selbst in dem, was man tut, verlieren), Interesse (Hingabe in der kreativen Tätigkeit selbst, nicht in ihrem Ergebnis), Neugier (,Balance von Sicherheit und Veränderungslust““ [ebd., 52]). 
äußert sich beispielsweise in der vermehrten Popularität sog. Kreativitätstrainings (vgl. kritisch hierzu Bröckling 2004).

Nach dem humanistischen Bildungsideal (vgl. von Humboldt 1792/1793) gelingt es dem Menschen durch Bildung, sich der eigenen Verantwortung sich selbst und seiner Umwelt gegenüber sowie bestehender Zwänge bewusst zu werden (vgl. Heydorn 2004). Diese emanzipatorische Haltung ermöglicht ihm Freiheit und Unabhängigkeit (vgl. von Humboldt 1792/1793; Liessmann 2006), Mündigkeit (vgl. Adorno 1971), Autonomie (vgl. Castoriadis 1991) und freiheitliche und demokratische Widerständigkeit gegen Ungerechtigkeiten und Diskriminierungen (vgl. Adorno 2006; Liessmann 2011; Krautz 2007b; Freire 1970). In diesem Sinne meint humanistische Bildung auch Bildung um der Erkenntnis willen und nicht unter Berücksichtigung ihrer (meist ökonomischen) Verwertbarkeit (vgl. Liessmann 2006).

Dies steht dem Einfluss des Neoliberalismus entgegen: Effizienz, Wettbewerbsfähigkeit und Ergebnisorientierung werden zu einem Merkmal von Bildungspolitik, Schulen werden ein Teil des nationalen Wirtschaftssystems und eine Produktionsschmiede sog. Humankapitals (vgl. Hakala/Uusikylä 2015). Ersichtlich ist dies etwa bei der Betonung der Eigenverantwortung im Lernen, was einerseits ein $\mathrm{Zu}$ gang zu Mündigkeit sein kann, andererseits strukturelle Einflussfaktoren auf und institutionelle Diskriminierung von Minoritäten entlang unterschiedlicher Intersektionalitätslinien (vgl. Crenshaw 1989) verdeckt (vgl. Gille 2013; Amabile 1983). Die Zeitoptimierung des Lernens (vgl. die Diskussion um Einschulungsalter, Abitur nach 12 oder 13 Schuljahren, Regelstudienzeiten) entbehrt der Muße, welcher Kreativität bedarf (vgl. Bröckling 2004), und erschwert eine vertiefte kritische Auseinandersetzung mit Lerninhalten. Das steht einer kritischen, Zeitabläufe verzögernden und notwendigen Widerständigkeit entgegen (vgl. Dörpinghaus 2007). Stattdessen wird Kreativität zur „Schlüsselkompetenz für die Zukunft“ (Braun 2011: 18) und zum Wettbewerbsvorteil erklärt. So schreibt Braun:

„Wenn man wüsste, was die Kreativität eines Menschen ausmacht, dann könnte man sie in Bildungs- und Erziehungsprozessen gezielt so fördern, dass eine Gesellschaft viele kreative Persönlichkeiten hervorbringen würde, welche ihr in der Folge dann eine Vorrangstellung in Wissenschaft und Kunst ermöglichen könnten.“ (Ebd., 13; vgl. auch Holm-Hadulla 2005)

Am konkreten Beispiel des Grundschulfachs Sachunterricht in Nordrhein-Westfalen zeigt sich eine neoliberal geprägte Bildung z.B. in der Verwendung von Schulbüchern, deren Aufgaben in der Regel lediglich zum Nachahmen, jedoch nicht zum eigenständigen, kritischen und kreativen Denken anregen (vgl. Weis 2016; Weis/Scharf/Gryl 2017). Die „Trends in International Mathematics and Science Study“ (TIMSS) verdeutlicht für den deutschen Mathematikunterricht, dass Inhalte 
komplexer Aufgaben oft unter starker Steuerung durch die Lehrperson in elementare Teilleistungen zerlegt werden (vgl. Klieme/Schümer/Knoll 2001). Die Lernenden arbeiten so nicht auf einer Ebene des Problemlösens, sondern auf einer der Reproduktion, Assoziation und der einfachen Operationen (vgl. ebd.). Auch sog. aktivierende Methoden zielen häufig vielmehr auf Beschäftigung mit einem Gegenstand, denn auf das Hinterfragen eines solchen ab (vgl. Gryl/Naumann 2016). Neoliberale (Kreativitäts-)Bildung fordert Originalität ein, jedoch innerhalb von Grenzen des Konformismus (vgl. Krautz 2007a), sodass Kreativität paradoxerweise gehemmt wird (vgl. Bröckling 2004).

Im Zuge der Entäußerung des Kreativitätsbegriffs von Widerständigkeit und schöpferischer Neugestaltung birgt eine neoliberale (Kreativitäts-)Bildung die Gefahr einer „Scheinpartizipation“ (Steenkamp 2017: 232) ${ }^{6}$., Reale Partizipation“ meint im Sinne von Weber et al. (2013), ,die aktive Mitwirkung von Menschen an all den Prozessen, die ihr Leben betreffen" (ebd., 9) und erfordert und fördert so Mündigkeit der Partizipierenden. Diese ist, angelehnt an Adorno (1971: 140ff.), die erlernbare Fähigkeit eines Menschen, die eigene Meinung zu sämtlichen Verhältnissen und Geschehnissen eigenständig und unabhängig zu bilden, diese kritisch zu reflektieren, angemessen zu artikulieren und nach dieser vernünftig und autonom zu handeln. ,Scheinpartizipation' meint hingegen die Engführung der Beteiligung innerhalb bestehender, die Optionen stark limitierender Strukturen, wodurch Partizipation lediglich vorgetäuscht wird (vgl. Hart 1992).

Für eine Bildungskritik ist es unabdingbar, die skizzierte neoliberale Vereinnahmung und Engführung des Kreativitätsbegriffs offenzulegen (vgl. Huber 2017). Eine allzu begriffspessimistische Perspektive auf Kreativität blendet jedoch aus, dass „,kulturelle Produktion selbst als Werkzeug gegen die kapitalistische Verwertung [verstanden und genutzt werden sollte]“ (ebd., 67) und Kreativität kritischem, gestalterischem Handeln sowie der Suche nach Alternativen innerhalb gesellschaftlicher Prozesse dienlich sein kann (vgl. Moegling 2017). Als Ausdruck von Widerständigkeit kann Kreativität so einen wichtigen Bestandteil einer humanistischen Bildung zur Mündigkeit darstellen.

6 ,Scheinpartizipation“ ist die gängige Übersetzung des Begriffs „Non-participation“ (Hart 1992: 9), welchen Hart (ebd.) verwendet, um die unteren Stufen der von ihm entworfenen Partizipationsleiter zu benennen - ein Stufenmodell, das unterschiedliche Grade der Beteiligung von Kindern und Jugendlichen beschreibt. 


\section{Bildung zur Innovativität}

Eine Herangehensweise zur Umsetzung stellt der Ansatz einer Bildung zur Innovativität ${ }^{7}$ dar. Dieser lehnt sich im Kontrast zur beschriebenen neoliberalen Praxis an humanistische Bildungsgestaltung (vgl. von Humboldt 1792/1793) zur Förderung von Mündigkeit und Partizipation an und beinhaltet ein humanistisches Kreativitätskonzept als zentrale Komponente der Fähigkeit des Innovierens (vgl. Gryl 2013; Jekel/Ferber/Stuppacher 2015; Weis et al. 2017). Innovativität wird als Fähigkeit betrachtet, welche die Suche nach Lösungen für die Gesellschaft umfasst, wie es Bröckling (2004) und Joas (1992) auch für Kreativität beschreiben. In diesem Sinne beinhaltet Innovativität reale Partizipationsfähigkeit: Nicht wie im kritisierten Kreativitätsimperativ soll jeder Mensch die Bürde tragen, kreativ sein zu müssen; mit einer Bildung zur Innovativität soll aber jedem Menschen ermöglicht werden, an der Gestaltung der Welt zu partizipieren, zu innovieren. ${ }^{8}$

In einem Modellentwurf zu Innovativität beschreiben Weis et al. (2017) diese als die Fähigkeit, an Innovationsprozessen zu partizipieren (vgl. Scharf/Weis/Gryl 2017). Diese Fähigkeit bedeutet das Hinterfragen, kritische Denken und selbstständige Handeln mit dem Ziel, sich gegen als problematisch empfundene gesellschaftliche Verhältnisse $\mathrm{zu}$ positionieren, Veränderung $\mathrm{zu}$ initiieren und Gesellschaft zu gestalten. In dem Modellentwurf in Anlehnung an Gryl (2013) sowie Jekel, Ferber und Stuppacher (2015) beinhaltet Innovativität die Komponenten ,Reflexivität', ,Kreativität' und ,Implementivität‘.

Gesellschaftliche Normen grenzen Vorstellbares ein (vgl. Popitz 2000). Hieraus ergibt sich die Notwendigkeit einer kritischen Auseinandersetzung mit Problemlagen der Welt (vgl. Popitz 2000) sowie dem Erkennen, Durchschauen und Infragestellen bestehender Werte und Regeln (vgl. Gryl 2013; Joas 1992) ${ }^{9}$. Diese müssen zwar nicht aufgegeben, können aber neu geordnet werden (vgl. Joas 1992). Der

7 Der Begriff ,Innovativität‘ teilt seine sprachliche Wurzel mit der des Innovationsbegriffs, welcher vorwiegend neoliberal konnotiert ist. Daher möchten wir an dieser Stelle darauf hinweisen, dass wir uns in erster Linie nicht auf wirtschaftswissenschaftliche Begriffsauslegungen (u.a. Maier et al. 2000) stützen, sondern auf sozialwissenschaftliche (u.a. Howaldt/Jacobsen 2010) und bildungswissenschaftliche (u.a. Rürup/Bormann 2013), welche den partizipationsfördernden Charakter des Begriffs betonen.

8 Vgl. Beuys' Aussage ,Jeder Mensch ein Künstler“, welche er auf der documenta 5 von 1972 erstmalig ausrief (vgl. Bröckling 2004). Nach dieser kann im Sinne eines ,erweiterten Kunstbegriffs“ jede*r die eigene Kreativität nutzen und an der Gesellschaft partizipieren (vgl. Schata 1976 sowie Beuys' Ausführungen zur ,Sozialen Plastik“ u.a. in Harlan/Rappmann/Schata 1976).

9 Vgl. hierzu auch Badious (2012) Ausführungen zum Konsens. 
Begriff ,Reflexivität' beschreibt diese Fähigkeit, bestehende Gegebenheiten und Routinen als Problemfelder zu identifizieren (vgl. Jekel/Ferber/Stuppacher 2015; Gryl 2013) und für Umweltveränderung und Handlung offen zu sein (vgl. Popitz 2000). Dabei bezieht der Reflexivitätsbegriff, im Gegensatz zum Begriff ,Reflexion', welcher externe Gegenstände hinterfragt, auch das eigene Denken (vgl. Schneider 2013) und Handeln (vgl. Luhmann 1998) ein (vgl. Gryl 2013). Reflexivität ist so eine notwendige Voraussetzung für Kreativität: „Creativity is our great need, but criticism, self-criticism is the way to its release“ (Dewey o.J., zit.n. Joas 1992: 8).

Kreativität wird im Modellentwurf zu Innovativität als die Fähigkeit zusammengefasst, Ideen zur Lösung identifizierter Probleme hervorzubringen (vgl. Weis et al. 2017; Jekel/Ferber/Stuppacher 2015; Gryl 2013), sodass der Ansatz einer Bildung zur Innovativität einen pragmatisch orientierten Kreativitätsbegriff (vgl. Braun 2011) $)^{10}$ umfasst. Das Kreativitätsverständnis dieses Ansatzes setzt sich aus Ausführungen von Popitz (2000) zu Kreativität ${ }^{11}$ zusammen, deren Komponenten von Kreativität und ihre Bezüge zueinander in der folgenden Abbildung illustriert werden.

10 Wenngleich wir uns in diesem Text kritisch zu Brauns (2011) neoliberalen Tendenzen bezüglich Kreativitätsförderung positionieren, erscheint uns ihre Unterscheidung zwischen ,ästhetischer' und ,pragmatischer Kreativität‘ hilfreich zur Beschreibung des Kreativitätskonzeptes des Ansatzes einer Bildung zur Innovativität. Während ,ästhetische Kreativität ' insbesondere bildnerisches, musisches und sprachliches Gestalten umfasst, bezeichnet ,pragmatische Kreativität‘ vor allem Problemlösung und Ideenfindung (vgl. ebd.).

11 Das Kreativitätsverständnis des Ansatzes einer Bildung zur Innovativität basiert insbesondere auf den im Fließtext beschriebenen Ausführungen zu Kreativität von Popitz (2000). Obschon wir seine Ausführungen zu Vorstellungskraft und Fantasie für den Ansatz einer Bildung zur Innovativität verwenden, können wir seine Ansichten zur Kreativität nicht in Gänze teilen: Ihm zufolge kann man nur dann kreativ sein, wenn man „überzeugt ist, das Nötige sei schon vorhanden“ (ebd., 125). Er erläutert dies mit seiner Beschreibung von ,sinnlosen ‘ Erfindungen, welche nur in einer gesättigten Gesellschaft möglich seien. Zum einen diskreditiert die Bezeichnung einer Gesellschaft als ,gesättigt ‘ jegliche Diskussionen um fehlende Gleichstellung von Minderheiten; zum anderen ist unklar, welche Erfindungen als sinnlos gelten, wenn z.B. Marcel Duchamps ReadyMades zwar keine direkt sichtbare Funktion haben, durch die Zweckentfremdung jedoch eine Kritik am bürgerlichen Kunstverständnis enthalten. Der Ansatz einer Bildung zur Innovativität sieht im Sinne einer Reflexivität hingegen gerade in dem Infragestellen bestehender Strukturen das zur Kreativität leitende Moment. 


\section{Abbildung 1: Differenzierungen bei Popitz' (2000) Kreativitätskonzept}

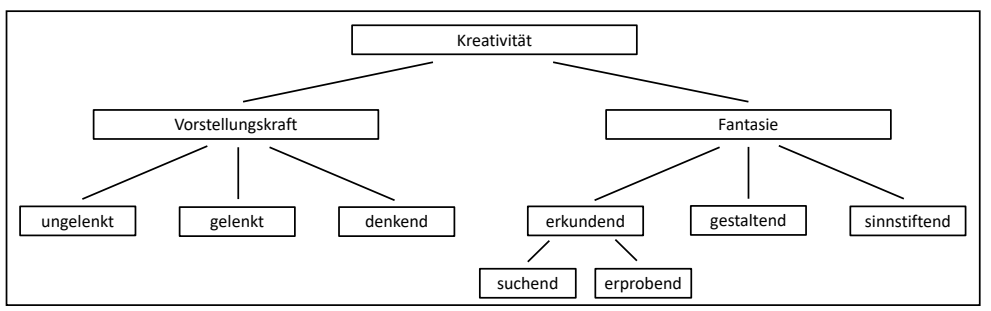

Quelle: Eigene Darstellung

Popitz (2000) beschreibt Kreativität als die Begabung zur Allozentrik, d.h. als Fähigkeit, die Welt in dem zu begreifen, was außerhalb des eigenen Selbst liegt. ,Vorstellungskraft" und ,Fantasie' bilden Bestandteile von Kreativität, wobei die Grenzen zwischen diesen beiden fließend sind (vgl. ebd.). Während mithilfe von Vorstellungskraft das zeitlich und/oder räumlich Abwesende vergegenwärtigt wird (vgl. ebd.), ermöglicht die Fantasie, noch nicht Existentes zu denken (vgl. ebd.; Bröckling 2004). Das heißt, dass Vorstellungskraft tatsächlich existente Gegenwirklichkeiten denken und auf eine andere Situation übertragen lässt und Fantasie das Imaginieren noch unbekannter Gegenwirklichkeiten ermöglicht (vgl. Popitz 2000). „Ungelenkte Vorstellungen“ (ebd., 88) in Form von unkontrollierten Bilderströmen oder einem Sich-treiben-lassen können ,gelenkte Vorstellungen“(ebd.), d.h. ein gezieltes Imaginieren von Alternativen, ermöglichen (vgl. ebd.). Diese Wechselbeziehung bedeutet für eine Bildung zur Innovativität, dass nicht nur gelenkte, sondern auch ungelenkte Vorstellungen einer Förderung bedürfen. „[D]enkende[...] Vorstellungen" (ebd.) bedeuten eine erste (innere) Artikulation der Gedanken (vgl. ebd.).

Auch bei der Fantasie nimmt Popitz (2000) Differenzierungen vor, welche das Kreativitätskonzept des Ansatzes einer Bildung zur Innovativität unterstützen: Er unterscheidet zwischen „erkundende[r]“ (ebd., 93) und „gestaltende[r] Fantasie“ (ebd. ${ }^{12}$, wobei er die erkundende Fantasie wiederum in suchende und erprobende Fantasie unterteilt (vgl. ebd., 104). Erstere beschreibt das bloße Erkunden, bei dem man ungeleitet auf Problemlösungen stoßen kann (vgl. ebd.), wodurch unserer Ansicht nach eine Analogie zu ungelenkten Vorstellungen besteht. Erprobende Fan-

12 Eine dritte Differenzierung von Fantasie sieht Popitz (2000) in der sinnstiftenden Fantasie. Diese interpretieren wir als die weltanschauliche Basis des Fantasierten. In dieser Lesart erscheint uns diese dritte Differenzierung bzw. die Möglichkeit einer ethischen Metaebene als noch zu erörternde Komponente im Ansatz einer Bildung für Innovativität, wie im letzten Abschnitt dieses Artikels angedeutet wird. 
tasie ist hingegen bereits eine imaginierte Handlung, nämlich das Abwägen von Möglichkeiten, um gezielt Problemlösungen zu finden (vgl. ebd.). Auch bezüglich der gestaltenden Fantasie nehmen wir insofern eine Analogie zu denkenden Vorstellungen wahr, als Popitz die gestaltende Fantasie als objektbildend und darstellend, d.h. als die Vergegenständlichung der eigenen Intention beschreibt.

Implementivität ist die Fähigkeit zu argumentieren und andere von dem Vorhandensein von Problemfeldern und/oder Lösungsideen zu überzeugen (vgl. Weis et al. 2017; Jekel/Ferber/Stuppacher 2015; Gryl 2013). In dem Modellentwurf zu Innovativität werden Popitz' (2000) denkende Vorstellungen als Konkretisierung und Artikulierung von Ideen als erste Vorstufe von Implementivität verstanden. Die erste innere Artikulation der eigenen Gedanken ist notwendig, um Formulierungen zu finden, mit denen andere von (der) Idee(n) überzeugt werden können. Dieser Schritt ist erforderlich, um die Welt tatsächlich und nicht nur rein theoretisch gestalten zu können: „Ob etwas kreativ ist oder nicht, zeigt sich erst im Nachhinein, wenn es anderen gefällt, einleuchtet oder brauchbar erscheint [...]. Einfach nur andere Wege zu gehen als die Masse, nützt gar nichts, solange sich niemand dafür interessiert“ (Bröckling 2004: 239; vgl. auch Sternberg/Lubart 1991). Um die Möglichkeit zu haben, die eigenen Gedanken und Ideen anderen gegenüber sichtbar zu machen, werden Ressourcen wie monetäre Mittel, rhetorische Fähigkeiten, Unterstützung durch andere oder bestimmte Fertigkeiten z.B. zur Umsetzung einer Idee benötigt.

Der Modellentwurf zu Innovativität (vgl. Weis et al. 2017) beschreibt neben den drei Komponenten Reflexivität, Kreativität und Implementivität auch ihre Wirksamkeit innerhalb von Innovationsprozessen. Dem Entwurf zufolge setzen sich Innovationsprozesse aus drei Phasen zusammen: ,Problemfelder identifizieren', ,Lösungen entwickeln“ und ,Lösungen implementieren' (vgl. ebd.). Wenngleich diese Unterscheidung zwischen Innovativität und Innovationsprozess zunächst tautologisch erscheint, ist diese insofern sinnvoll, als sie die Verwobenheit der beschriebenen Komponenten von Innovativität miteinander thematisiert und diese nicht auf je eine Phase eines Innovationsprozesses festlegt.

Scharf, Schmitz und Gryl (2016) zufolge kann eine Beteiligung an Innovationsprozessen sowohl aktiv als auch reaktiv erfolgen (vgl. auch Hartmann/MeyerWölfing 2003). Ersteres meint das aktive Identifizieren von Problemlagen, Ideen und deren Implementierung (vgl. Scharf/Schmitz/Gryl 2016). Letzteres beschreibt hingegen das Reagieren auf identifizierte Problemlagen und darauf basierende Implementierungsversuche (vgl. ebd. sowie Weis 2016). Somit sind Innovationsprozesse geprägt von Rückkopplungsschleifen und dadurch immer kollaborativ (vgl. Scharf et al. 2018 sowie bezogen auf Kreativität Bröckling 2004).

Hinsichtlich der im Modellentwurf zu Innovativität aufgeführten Komponenten bedeutet das, dass am Innovationsprozess beteiligte Personen nicht nur während 
der Identifizierung von Problemfeldern bestehende Strukturen im Sinne der Reflexivität hinterfragen, sondern auch in anderen Phasen von Innovationsprozessen. Das ist der Fall, wenn es um das ungelenkte, gelenkte und denkende Infragestellen eigener Lösungsideen und Umsetzungsstrategien wie auch Ideen und Strategien anderer geht. Ebenso spielt Kreativität in allen Phasen eines Innovationsprozesses eine Rolle (vgl. Scharf/Weis/Gryl 2017): Im Sinne von Popitz' (2000) Ausführungen ist die erkundend suchende Fantasie für das aktive Infragestellen bestehender Gegebenheiten von Bedeutung. Dies gilt auch für die erkundend erprobende Fantasie, welche außerdem für die Vorbereitung von Entwicklungen von Lösungsideen notwendig ist, die wiederum mit denkender Vorstellungskraft und gestaltender Fantasie realisiert und kreativ implementiert werden. Implementivität ist nicht nur eine notwendige Fähigkeit, wenn andere von einer Lösungsidee überzeugt werden sollen, sondern auch bei der Thematisierung von Problemfeldern. Diese Verwobenheit und die damit einhergehende Möglichkeit, Innovationsprozesse jederzeit zu betreten und zu verlassen, betont deren Dynamik (vgl. Weis et al. 2017) im Gegensatz zu linear aufgebauten Beschreibungen von Innovationsprozessen (vgl. u.a. Vahs/Burmester 1999).

\section{Innovativität in der Praxis der schulischen Bildung}

Aus diesen theoretischen Ausführungen lassen sich Überlegungen zu einer Bildung zur Innovativität im schulischen Kontext ableiten. Das Grundschulfach ,Sachunterricht' stellt hier einen möglichen Rahmen dar, da Curricula (vgl. u.a. MSW NRW 2008) wie auch die Gesellschaft für die Didaktik des Sachunterrichts (vgl. GDSU 2013) dem Fach eine mündigkeitsbildende Zielsetzung zuschreiben, was eine Förderung von Innovativität nahelegt.

Im Kontext der schulischen Bildung scheinen sich Schüler*innen Innovativität am ehesten in offenen Lehr-Lernsituationen aneignen zu können (vgl. Weis 2016; Weis/Scharf/Gryl 2017). Das heißt auch, dass Innovativität, den von von Osten (2003) kritisierten Kreativitätsimperativ kontrastierend, nicht angeordnet werden soll, z.B. in Form einer in sich geschlossenen Unterrichtseinheit. Vielmehr soll stets der Freiraum zu eigenem Denken und kritischer Auseinandersetzung geboten werden: „Kreativitätsförderung [im Sinne von Innovativitätsförderung, die Autor*innen] ist Kontextsteuerung; sie schafft nichts, sie ermöglicht" (Bröckling 2004: 240). Ideen sollen zwar kritisch hinterfragt und so kollaborativ elaboriert, jedoch nicht durch vermeintlich realitätsnahe Aussagen eingegrenzt und in Abrede gestellt werden. Eine Benotung von Reflexionen, Ideen und Umsetzungsstrategien würde einer Bildung zur Innovativität ebenso im Wege stehen, da sie Reflexivität insofern eingrenzt, als die benotende Person bzw. deren Maßstäbe als Basis für Reflexionen, Ideen und Umsetzungsstrategien das Infragestellen eben jener Maßstäbe 
erschwert. Zumal ein Erwartungshorizont zur Bewertung des Innovierens die Idee von Innovativität insofern konterkariert, als durch ihn Reflexion, Idee und Umsetzungsstrategie bereits vorweggenommen und auf eine Variante reduziert würden.

Einen Ansatz der Umsetzung einer Bildung zur Innovativität stellen theoretische Überlegungen und erste explorative Untersuchungen zur didaktischen Methode der Simulation dar (vgl. Weis et al. 2017, auf Basis von Überlegungen zu Planspielen von Rebmann 2001 und Geuting 1992). Hierbei haben Schüler*innen in simulierten Szenarien innerhalb fiktiver Weltentwürfe an Innovationsprozessen teil, welche thematisch an ihre Lebenswelt anknüpfen, indem sie sich mit vorgegebenen Problemfeldern auseinandersetzen (vgl. ebd.). Weis et al. (2017) vermuten, dass der Einsatz von Simulationen einer Bildung zur Innovativität dienlich sein kann, da sie mit variablen Themenfeldern durchgeführt werden können und somit nicht an eine Unterrichtseinheit gebunden sind. Ferner können Zukunftsgesellschaften losgelöst von aktuellen Einschränkungen gedacht werden (vgl. ebd.). Der offene Lehr-Lernraum dieser Methode ermöglicht zudem eine freie und kreative Auseinandersetzung mit den behandelten Themen (vgl. ebd.).

Innerhalb eines Workshopsettings im Kontext der Erwachsenenbildung haben Weis et al. (2017) eine erste explorative Vorstudie zu diesen Überlegungen durchgeführt, um zu erkunden, inwieweit diese Methode einer Bildung zur Innovativität zuträglich sein kann. Hierbei wurden die Workshopteilnehmenden, ohne darüber im Vorfeld informiert worden zu sein, zunächst als Mitglieder eines Bildungsministeriums eines fiktiven Staates begrüßt, deren Aufgabe nun darin bestehe, das Bildungssystem zu erneuern (vgl. ebd.). In Gruppenarbeit entwickelten sie anschließend Entwürfe, diskutierten diese im Plenum und stimmten für den Entwurf $\mathrm{ab}$, den sie als beste Idee einstuften. Auf einer Metaebene wurde abschließend die Simulationsmethode reflektiert, u.a. indem der zugrundeliegende Modellentwurf zu Innovativität präsentiert wurde (vgl. ebd.).

Die vorläufigen Forschungsergebnisse dieser Simulation zeigen, dass diese Methode das Potenzial besitzt, die Innovativität der Teilnehmenden zu fördern: Durch die Loslösung von realen Restriktionen konnten sie Ideen entwickeln, welche in der Realität nicht möglich wären. Das Vorhandensein dieser Ideen kann in einem weiteren Schritt unter Umständen die Teilnehmenden in die Lage versetzen, jene Gegebenheiten, die diese Ideen gewöhnlich verhindern, infrage zu stellen. Ein neoliberales, konkurrenzbasiertes Verständnis von Lehr-Lernarrangements scheint hingegen Innovativität zu hemmen: Faktoren wie ein ausreichendes Zeitfenster, gestalterischer Freiraum (etwa in Form offener Aufgabenformate oder eines bewertungsfreien Kontextes) sowie ein wohlwollendes, positives Arbeitsklima scheinen die Innovativität der Teilnehmenden anzuregen. 


\section{Abschluss und Ausblick}

Während eine neoliberale Auslegung des Kreativitätsbegriffs, insbesondere im Bildungssektor, Menschen in ihrer Mündigkeit beschneidet, kann die Förderung von Kreativität in einer humanistischen Lesart emanzipatorische Denk- und Handlungsweisen ermöglichen. Ein solches Kreativitätsverständnis stellt einen wichtigen Bestandteil des Ansatzes einer Bildung zur Innovativität dar, welcher Schüler*innen zur Partizipation befähigen möchte. Zugleich betont der Ansatz die Notwendigkeit von Reflexivität, also der Fähigkeit zu kritischem Hinterfragen, sowie Implementivität, d.h. der Argumentationsfähigkeit zur Verbreitung und möglichen Umsetzung von Ideen für die Gestaltung von Gesellschaft. Diese Einbettung hebt das Konzept der Kreativität in den Anwendungskontext der Partizipationsfähigkeit. Angesichts der Verankerung von Reflexivität und der Option auf Veränderung ist jene Partizipation eine mündige. Der Ansatz einer Bildung zur Innovativität ist jedoch noch nicht vollständig elaboriert. Neben der hier vertieften Auseinandersetzung mit Kreativität bedarf es weiterer theoretischer Vertiefung und empirischer Erprobung.

Trotz der humanistischen Zielsetzung des Ansatzes einer Bildung zur Innovativität birgt dieser die Gefahr, neoliberale Bildungsformen zu unterstützen: Die Person, die über die meisten Ressourcen, z.B. eine Machtposition in einem Netzwerk, verfügt, kann sich am leichtesten Gehör verschaffen und so andere mit vergleichsweise geringem Aufwand von ihren Ideen überzeugen (vgl. die Kategorie ,Einfluss` des Machtbegriffs von Olsen/Marger 1993, zit.n. Imbusch 2012). Insbesondere massentaugliche Ideen würden so die meiste Aufmerksamkeit erregen und am leichtesten implementiert werden, auch wenn diese nicht im Einklang mit einer reflexiven und widerständigen Auseinandersetzung mit jener Masse stehen mögen (vgl. auch Joas' [1992] Ausführungen zu Partizipation und ,Yuppisierung').

Ferner erscheint eine ethische Metaebene des Ansatzes einer Bildung zur Innovativität notwendig. Der Ansatz beinhaltet zwar bereits durch die Komponente des reaktiven Innovierens prinzipiell die Option der Nicht-Unterstützung einer Idee, dennoch würden ohne ethische Metaebene fragwürdige Neuerungen als Endprodukt eines Innovationsprozesses und eine Teilnahme an solchen Prozessen als wünschenswert und förderlich angesehen werden können. Wenn die im Modellentwurf beschriebene Reflexivität Allozentrik als Auseinandersetzung mit Problemlagen der Welt (vgl. Popitz 2000) beinhaltet, ist zu fragen, welche Problemlagen als solche anerkannt sind und welche nicht und wer die Deutungsmacht in Fragen der Sinnstiftung (vgl. ebd.) trägt. Eine ethische Metaebene als Bestandteil des Modellentwurfs zu Innovativität erscheint daher sinnvoll. Mögliche Grundlagen dieser ethischen Metaebene können neben (Angewandter) Ethik die Kritische Theorie und Kritische Pädagogik sowie Friedensforschung und Friedenspädagogik bilden. 
Neben der Fortentwicklung des Modellentwurfs zu Innovativität sind bestehende Wege auszuloten und neue Methoden zu entwickeln, mit denen Innovativität (im schulischen Kontext) gefördert werden kann. Das beinhaltet auch die Weiterentwicklung der Methode der Simulationen, welcher, obgleich sie eine Bildung zur Innovativität gegebenenfalls ermöglicht, in der bisherigen Konzeption Grenzen gesetzt sind. Da zum einen die Vorgabe eines Problemfeldes die Problemfindungsphase vom Innovationsprozess trennt, sollen in der Weiterentwicklung dieser Methode die behandelten Themen nicht mehr bestimmt werden. Der Zwang, sich nach Abschluss der Gruppenarbeits- und Ideenfindungsphase für einen Vorschlag zu entscheiden, kann zum anderen dazu führen, dass der Innovationsprozess abrupt und ohne notwendige Rückkopplungsschleifen abgeschlossen wird. Daher soll die Entscheidungsfindung am Ende der Methode offener gestaltet werden. Wie in dieser Methode bereits in Teilen realisiert, mag bei der (Weiter-)Entwicklung von innovativitätsfördernden Methoden eine Vertiefung von spielerischen und gestalterischen Tätigkeiten dienlich sein: Nach Popitz (2000) ist Spielen ein zwangloser Ausdruck der Verknüpfung der drei Fantasie-Wege (erkunden, gestalten, sinnstiften): „In der flüchtigen Kreativität des Spielens erfinden wir , unrealistische Realitäten“, etwas Reales, das zugleich fiktiv ist, eine Als-ob-Realität"“ (ebd., 97). So kann die Verbindung von (scheinbar) zweckfreiem und schöpferischem Handeln (vgl. Bröckling 2004 bezugnehmend auf Joas 1992) ,etwas Dauerhaftes inspirieren“ (Popitz 2000: 97). Ein Fokus auf ästhetische Bildung erscheint im Rahmen einer Bildung zur Innovativität aufgrund der Wechselwirkung von pragmatischer und ästhetischer Kreativität (vgl. Braun 2011) sinnvoll. Gestaltende Tätigkeiten ermöglichen Kindern, Bestehendes zu verändern und Neues zu schaffen (vgl. ebd.). Dies impliziert auch, ,kreative Problemlösungen für bestimmte Ausdrucksintentionen zu finden“" (ebd., 26), wie Studien zu Vermittlungsansätzen in der ästhetischen Bildung (vgl. u.a. Bamford 2006; „Learning Through Art“) belegen möchten (vgl. Braun 2011).

\section{LITERATUR}

Adorno, Theodor W. (1971): Erziehung zur Mündigkeit. Vorträge und Gespräche mit Hellmut Becker 1959 bis 1969, Frankfurt a.M.: Suhrkamp.

Ders. (2006): Theorie der Halbbildung [1959], Frankfurt a.M.: Suhrkamp.

Althusser, Louis (2010): Ideologie und ideologische Staatsapparate, (frz. 1970), Hamburg: VSA.

Amabile, Teresa M. (1983): „Social Psychology of Creativity. A Componential Conceptualization“, in: Journal of Personality and Social Psychology 45, S. 997-1013. 
Badiou, Alain (2012): „Das politische Feld heute. Die Opposition links/rechts, der Konsens“, in: Ders./Fabien Tarby (Hg.), Die Philosophie und das Ereignis, Wien: Turia + Kant, S. 9-48.

Bamford, Anne (2006): The wow factor: Global research compendium on the impact of the arts in education, Münster: Waxmann.

Boas, Taylor C./Gans-Morse, Jordan (2009): „Neoliberalism. From New Liberal Philosophy to Anti-Liberal Slogan“, in: Studies in Comparative International Development 44, H. 2, S. 137-161.

Braun, Daniela (2011): Kreativität in Theorie und Praxis. Bildungsförderung in Kita und Kindergarten, Freiburg/Basel/Wien: Herder.

Bröckling, Ulrich (2004): „Über Kreativität. Ein Brainstorming“, in Ders./Axel Paul/Stefan Kaufmann (Hg.), Vernunft - Entwicklung - Leben. Schlüsselbegriffe der Moderne, München: Wilhelm Fink, S. 235-244.

Castoriadis, Cornelius (1991): Philosophy, Politics, Autonomy, New York/Oxford: Oxford University Press.

Cornell University/INSEAD/WIPO (2018): The Global Innovation Index 2018: Energizing the World with Innovation, Ithaca/Fontainebleau/Geneva: WIPO.

Crenshaw, Kimberlé (1989): „Demarginalizing the Intersection of Race and Sex. A Black Feminist Critique of Antidiscrimination Doctrine, Feminist Theory and Antiracist Policy“, in: University of Chicago Legal Forum 1, H. 8, S. 139 167.

Dörpinghaus, Andreas (2007): „Bildungszeiten. Über Bildungs- und Zeitpraktiken in der Wissensgesellschaft", in: Hans-Rüdiger Müller/Wassilios Stravoravdis (Hg.), Bildung im Horizont der Wissensgesellschaft, Wiesbaden: Springer VS, S. $35-47$.

Freire, Paulo (1970): „Politische Alphabetisierung. Einführung ins Konzept einer humanisierenden Bildung“, in: Ders., Unterdrückung und Befreiung, Münster u.a.: Waxmann, S. 27-43.

GDSU (Gesellschaft für Didaktik des Sachunterrichts) (Hg.) (2013): Perspektivrahmen Sachunterricht, Regensburg: Klinkhardt.

Geuting, Manfred (1992): Planspiel und soziale Simulation im Bildungsbereich, Frankfurt a.M.: Peter Mann.

Gille, Annette (2013): „Die Ökonomisierung von Bildung und Bildungsprozessen aus dispositivanalytischer Sicht", in: Johanna Carbon Wengler/Britta Hoffarth/Lukasz Kumiega (Hg.), Verortungen des Dispositiv-Begriffs. Analytische Einsätze zu Raum, Bildung, Politik, Wiesbaden: Springer Fachmedien, S. 73-89.

Gryl, Inga (2013): „Alles neu. Innovation durch Geographie und GW-Unterricht?“, in: $G W$-Unterricht 131, H. 3, S. 16-27.

Dies./Naumann, Jasmin (2016): „Mündigkeit im Zeitalter des ökonomischen Selbst?“, in: GW-Unterricht 141, H. 1, S. 19-30. 
Hakala, Juha/Uusikylä, Kari (2015): „Neoliberalism, Curriculum Development and Manifestations of ,Creativity““, in: Improving Schools 18, H. 3, S. 250-262.

Harlan, Volker/Rappmann, Rainer/Schata, Peter (1976) (Hg.): Soziale Plastik. Materialien zu Joseph Beuys, Achberg: Achberger.

Hart, Roger (1992): Children's Participation: From Tokenism to Citizenship, UNICEF Innocenti Essays, Nr. 4, Florenz: International Child Development Centre of UNICEF.

Hartmann, Thomas/Meyer-Wölfing, Erwin (2003): „Nutzung von Innovationspotentialen in außerbetrieblichen Handlungs- und Lernfeldern“, in: QUEM-Report 83, 3-127.

Heydorn, Heinz-Joachim (2004): Über den Widerspruch von Bildung und Herrschaft [1970], Wetzlar: Büchse der Pandora.

Holm-Hadulla, Rainer M. (2005): Kreativität. Konzept und Lebensstil, Göttingen: Vandenhoeck \& Ruprecht.

Howaldt, Jürgen/Jacobsen, Heike (Hg.) (2010): Soziale Innovation. Auf dem Weg zu einem postindustriellen Innovationsparadigma, Wiesbaden: Springer VS.

Huber, Laila Lucie (2017): Kreativität und Teilhabe in der Stadt. Initiativen zwischen Kunst und Politik in Salzburg, Bielefeld: transcript.

Humboldt, Wilhelm von (1792/1793): „,Theorie der Bildung des Menschen (Bruchstück)“, in: Andreas Flitner/Klaus Giel (Hg.) (1969), Wilhelm von Humboldt, Darmstadt: Cotta, S. 234-240.

Imbusch, Peter (2012): „Macht und Herrschaft in der wissenschaftlichen Kontroverse“, in: Ders. (Hg.), Macht und Herrschaft. Sozialwissenschaftliche Theorien und Konzeptionen, Wiesbaden: Springer VS, S. 9-36.

Jekel, Thomas/Ferber, Nicole/Stuppacher, Kirstin (2015): „Innovation vs. Innovativeness. Do We Support Our Students in (Re-)Inventing the World?“, in: GI_Forum 3, S. 373-381.

Joas, Hans (1992): Die Kreativität des Handelns, Frankfurt a.M.: Suhrkamp.

Klieme, Eckhard/Schümer, Gundel/Knoll, Steffen (2001): „Mathematikunterricht in der Sekundarstufe I: ,Aufgabenkultur' und Unterrichtsgestaltung“", in Eckhard Klieme/Jürgen Baumert (Hg.), TIMSS - Impulse für Unterricht und Schule, Bonn: Bundesministerium für Bildung und Forschung, S. 43-57.

Krautz, Jochen (2007a): Ware Bildung. Schule und Universität unter dem Diktat der Ökonomie, Kreuzlingen/München: Hugendubel.

Ders. (2007b): „Pädagogik unter dem Druck der Ökonomisierung. Zum Hintergrund von Standards, Kompetenzen und Modulen“, in: Pädagogische Rundschau 1, S. 81-93.

„Learning through art“, https://www.guggenheim.org/for-educators/learningthrough-art 
Liessmann, Konrad Paul (2006): Theorie der Unbildung. Die Irrtümer der Wissensgesellschaft, Wien: Paul Zsolnay.

Ders. (2011): „Die letzte Aufgabe unseres Daseins. Über Bildung und ihre Deformation im Zeitalter des Wissens", in: Marisol Sandoval et al. (Hg.), Bildung MACHT Gesellschaft, Münster: Westfälisches Dampfboot, S. 39-50.

Luhmann, Niklas (1998): Die Wissenschaft der Gesellschaft, Frankfurt a.M.: Suhrkamp.

Macedo, Donaldo (2006): Literacies of Power: What Americans are not Allowed to Know With New Commentary by Shirley Steinberg, Joe Kincheloe, and Peter McLaren, Abingdon-on-Thames: Routledge.

Maier, Günter W./Frey, Dieter/Schulz-Hardt, Stefan/Brodbeck, Felix C. (2000): „Innovation“, in: Gerd Wenninger (Hg.), Lexikon der Psychologie, Heidelberg: Spektrum.

Moegling, Klaus (2017): Kultureller Transfer und Bildungsinnovation: Wie Schulen die nächste Generation auf die Zukunft der Globalisierung vorbereiten können, Kassel: Prolog.

MSW NRW (Ministerium für Schule und Weiterbildung des Landes NordrheinWestfalen) (Hg.) (2008): Richtlinien und Lehrpläne für die Grundschule in Nordrhein-Westfalen, Frechen: Ritterbach.

von Osten, Marion (2003): „Be Creative! Der kreative Imperativ“, in: Dies. (Hg.), Norm der Abweichung, Wien/New York: Springer, S. 159-210.

Popitz, Heinrich (2000): Wege der Kreativität, Tübingen: J.C.B. Mohr (Paul Siebeck).

Raunig, Gerald/Wuggenig, Ulf (2007): „Kritik der Kreativität. Vorbemerkungen zur erfolgreichen Wiederaufnahme des Stücks Kreativität‘, in: Dies. (Hg.), Kritik der Kreativität, Wien: Turia + Kant, S. 9-14.

Rebmann, Karin (2001): Planspiel und Planspieleinsatz, Hamburg: Dr. Kovac.

„Resilienz \& neoliberale ,Eigenverantwortung““”, https://www.medico.de/resilienz-neoliberale-eigenverantwortung-15984/

Rürup, Matthias/Bormann, Inka (Hg.) (2013): Innovationen im Bildungswesen. Analytische Zugänge und empirische Befunde, Wiesbaden: Springer VS.

Scharf, Claudia/Gryl, Inga/Gamper, Markus/Weis, Swantje (2018): „Collaborative Innovating - The Potential of Street Art to Shape Spaces“, in: GI_Forum 2, S. 156-167.

Scharf, Claudia/Schmitz, Stephan/Gryl, Inga (2016): „Innovativeness as Fresh Ground. From an Old Buzzword to New Praxis“, in: GI_Forum 1, S. 250-261. Scharf, Claudia/Weis, Swantje/Gryl, Inga (2017): „Innovative Pupils. Documentary Research on Teaching and Learning Arrangements for Innovativeness“, in: The European Conference on Education: Official Conference Proceedings, S. 295313. 
Schata, Peter (1976): „Das Oeuvre des Joseph Beuys. Ein individueller Ansatz zu universeller Neugestaltung“،, in: Harlan/Rappmann/Schata (Hg.), Soziale Plastik. Materialien zu Joseph Beuys, Achberg: Achberger, S. 75-120.

Schneider, Antje (2013): Geographiedidaktische Reflexivitat. Ostdeutsche Mobilitatsfragen im zweiten Blick, Berlin: LIT.

Schumpeter, Joseph (2005): Kapitalismus, Sozialismus und Demokratie, (engl. 1947), Tübingen u.a.: Francke.

Sennett, Richard (1998): Der flexible Mensch. Die Kultur des neuen Kapitalismus, (engl. 1998), Berlin: Berlin-Verlag.

Springer, Simon/Birch, Kean/MacLeavy, Julie (2016): „Introduction to Neoliberalism“, in: Dies. (Hg.), The Handbook of Neoliberalism, New York: Routledge, S. 1-13.

Steenkamp, Daniela (2017): „Für mehr Beteiligung von Kindern in der Schule Wie Kinder online Demokratie lernen können”, in: Dies./Margit Stein (Hg.), Kinderrechte sind Menschenrechte. Stand, Perspektiven und Herausforderungen, Berlin: LIT, S. 231-236.

Sternberg, Robert/Lubart, Todd (1991): „An Investment Theory of Creativity and its Development", in: Human Development 34, S. 1-31.

Vahs, Dietmar/Burmester, Ralf(1999): Innovationsmanagement. Von der Produktidee zur erfolgreichen Vermarktung, Stuttgart: Schäffer-Poeschel.

Weber, Susanne Maria/Göhlich, Michael/Schröer, Andreas/Macha, Hildegard/Fahrenwald, Claudia (2013): „Organisation und Partizipation - interdisziplinäre Verhältnisbestimmungen und organisationspädagogische Perspektiven“, in: Susanne Weber (Hg.), Organisation und Partizipation. Beiträge der Kommission Organisationspädagogik, Wiesbaden: Springer VS, S. 9-28.

Weis, Swantje (2016): Schüler als Entdecker, Erfinder, Erneuerer?, Unveröffentlichte Staatsarbeit, Essen.

Dies./Scharf, Claudia/Gryl, Inga (2017): „New and Even Newer. Fostering Innovativeness in Primary Education“, in: IJAEDU 3, H. 7, S. 209-219.

Weis, Swantje/Scharf, Claudia/Greifzu, Lena/Gryl, Inga (2017): „Stimulating by Simulating. Fostering Innovativeness in Education", in: International Conference on Education: IACB, ICE \& ICTE Conference Proceedings, S. 386/1$386 / 11$.

„Weltweiter Vergleich: Deutschland ist am innovativsten“,

https://www.tagesschau.de/wirtschaft/innovation-deutschland-101.html vom 17.10.2018.

Žižek, Slavoj (2012): „Living in the Time of Monsters“, in: Maria Nikolakaki (Hg.), Critical Pedagogy in the New Dark Ages, New York: Peter Lang, S. 3244. 


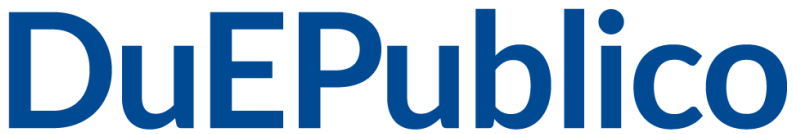

Duisburg-Essen Publications online offen im Denken

In: Kritische Kreativität: Perspektiven auf Arbeit, Bildung, Lifestyle und Kunst / Kim Kannler, Valeska Klug, Kristina Petzold, Franziska Schaaf (Hg.) transcript Verlag, Bielefeld, 2019 (S. 203-218)

Print-ISBN 978-3-8376-4619-1 - PDF-ISBN 978-3-8394-4619-5 - Book DOI: 10.14361/9783839446195

Dieser Text wird über DuEPublico, dem Dokumenten- und Publikationsserver der Universität Duisburg-Essen, zur Verfügung gestellt. Die hier veröffentlichte Version der EPublikation kann von einer eventuell ebenfalls veröffentlichten Verlagsversion abweichen.

DOI: $\quad 10.14361 / 9783839446195-011$

URN: urn:nbn:de:hbz:464-20200226-093040-6 